\title{
Evaluation of raw water quality in Wassit governorate by Canadian water quality index
}

\author{
Abdulkhaleq Mahmood \\ Water Resource Department, Kut Technical Institute, Middle Technical University, Kut, Iraq
}

\begin{abstract}
In this work an attempt has been done to evaluate the raw water quality in Wassit Governorate by using Canadian Council of Ministers of Environment Water Quality Index (CCME WAI). Six stations along Tigris River were located and the field work was conducted during one year from October 2015 to September 2016 in collecting data. Twelve water parameters were used to evaluate the water quality index $(\mathrm{pH}$, turbidity, total dissolved solid, total alkalinity, total hardens, nitrate, calcium, sodium, potassium, magnesium, chloride, and sulfate). The raw water quality in Wassite Governorate has been ranged between (65-79) based on CCME WQI results, which means that the river has not been in its good condition, and need to be manage to control the sources of pollutants by monitoring to keep them in their natural condition.
\end{abstract}

\section{Introduction}

One of the essential part of any environmental monitoring program is the reporting of results to both general public and the managers. This task is so complex in water quality monitoring because of the large number of measured variables required analyzing. One of the suggested solution of this problem is employing an (water quality index), that will arithmetically combine all water variable parameters measures and provide a general understood description of water. This index is a good tool for ranking the suitability of water use by humans, wild life, aquatic life, etc. [1]. Water quality index was creating by the Canadian Council of Ministries of Environment (CCME) in 1997. The index value can range between 0 and 100 . Increasing values are indicative of increasing water quality in which CCME WQI value between 95-100 means that the water quality is excellent while CCME WQI values between 0-44 means that the water quality is poor. In Iraq, many attempts has been done to evaluate the water quality index for both Tigris and Euphrates rivers in different locations. Shaymma and Ayad, 2012 studied the water quality index for Euphrates river from Al-Qaim to Al-Qurna for both drinking and irrigations water. The results shows that the water quality for overall drinking and irrigation uses in the period between April 1998 to April 2001 is mostly rated between good to very poor [2]. Alhassan, 2013 make an attempt to calculate the overall water quality index for
Tigris river as a case study using six water parameters in Baghdad city. The results indicated that the Tigris water quality are ranged between poor and slightly polluted [3]. Layla, 2015 used CCME QWI to evaluate the water quality index of three adjacent water treatment stations in Shat AlHila River. The results showed that all evaluated parameters of treated water were within Iraqi standard except turbidity and the water quality is good [4]. Salam, 2016 studied the water quality of Al-Gharraf river in south of Iraq using CCME WQI. The result shows that the water of Al-Gharraf classified to poor for aquatic life and fair for irrigation with seasonal overall WQI values of 30-39 and among stations was 38-29.

The objective of the present research is to evaluate the raw water quality of Tigris river in Wassit Governorate using CCME WQI.

\section{Data and samples collection}

To assess water quality conditions relative to water quality objectives, twelve water parameters were tested during one year from October 2015 to September 2016. Six locations were used for tacking samples over $160 \mathrm{~km}$ long on Tigris River within Wassite governorate. The sampling locations distributed between Al-Swiara and Al-Kut City. Water samples were tested twice in month and the average values for each parameters were recorded for WQI evaluation. 
Figure 1 shows the aerial photo for sampling location within Wassite governorate, while table 2.1

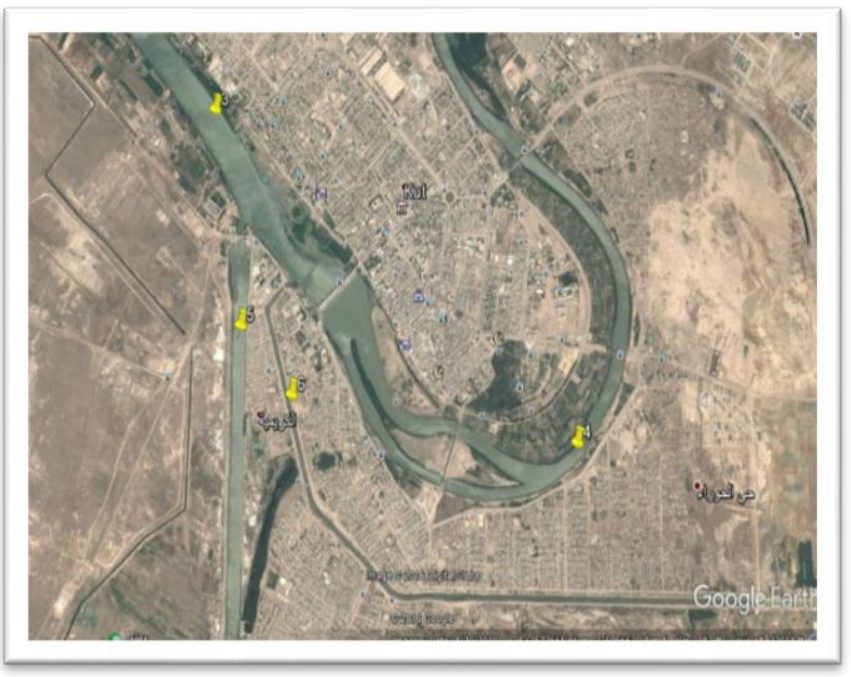

shows the UTM coordinate for sampling locations.

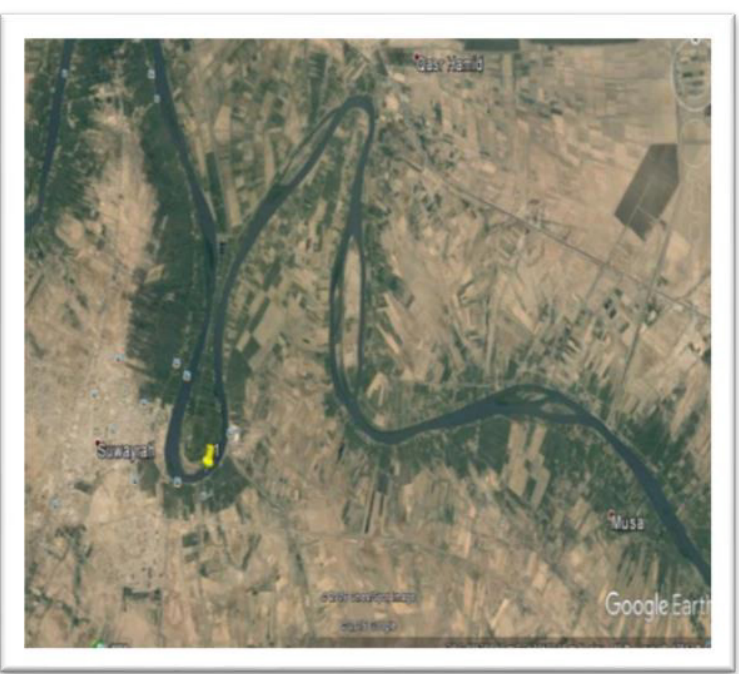

Fig. 1. Aerial photo for sampling locations within Wassite governorate.

Table 1. The UTM coordinates for sampling locations

\begin{tabular}{|c|c|c|}
\hline Sample no. & $\mathrm{N}$ & $\mathrm{E}$ \\
\hline 1 & 3642820 & 481467 \\
\hline 2 & 3602139 & 539368 \\
\hline 3 & 3597279 & 575568 \\
\hline 4 & 3594828 & 579097 \\
\hline 5 & 3595648 & 575885 \\
\hline 6 & 3595150 & 576391 \\
\hline
\end{tabular}

\subsection{Water quality index measurement}

The selection of appropriate water quality variables is necessary to yield meaningful water quality index results. This related to a professional judgment of the observer. After collecting the data for the twelve parameters for raw water in Wassite governorate during one years, the water quality index were calculated using CCEM WQI. The values of water quality index factors (F1, F2, and F3) are calculated as flow, F1, and F2 are calculated directly, while F3 are need some additional steps.

$$
\mathrm{F} 1=\frac{\text { Number of variables that failed }}{\text { Total number of variables }} * 100
$$

Where F1, is the percentage of variables that not meet the objectives at least one during the period of observation failed variables).

$$
\mathrm{F} 2=\frac{\text { Number of failed test }}{\text { Total number of tests }} * 100
$$

Where F2, is the percentage of tests that not meet the objectives (failed test). For calculation the factor F3, which represented by the equation

$$
\mathrm{F} 3=\frac{n s e}{0.01 \text { nse }+0.01}
$$

In which, F3 represent the amount by which failed tests values don't meet their objectives, and nse is the normalized sum of excursion, which are calculated by

$$
\text { nse }=\frac{\sum_{i=1}^{n} \text { excursion }}{\text { fof tests }}
$$


equation are calculated by dividing the sum of excursion that represented the amount by which each failed test are out of complains with relative of all tests( sum of tests meet and not meet objectives).

The excursion are calculated either by

$$
\text { Excursion }=\left(\frac{\text { test values failed }}{\text { objectives }}\right)-1
$$

This equation is used when the test values must not exceed the objectives.

$$
\text { Excursion }=\left(\frac{\text { objectives }}{\text { test values failed }}\right)-1
$$

This equation is used when the test values is fall below the objectives.

After calculating the three factors ( F1,F2, and F3) the

$$
\text { CCME Water Quality Index }=\frac{\sqrt{F 1^{2}+F 2^{2}+F 3^{2}}}{1.732}
$$

The CCME.WQI results ranged between 0 and 100 as shown in table 2.

Table 2. Shows the ranking of water categories due to CCME results

\begin{tabular}{|l|l|}
\hline Degree & Water categories \\
\hline $95-100$ & Excellent ( natural water, no threat detected) \\
\hline $80-94$ & Good ( rarely depart from natural condition) \\
\hline $65-79$ & Fair ( sometimes depart from natural levels) \\
\hline $45-64$ & Marginal ( often depart from natural and desirable level) \\
\hline $0-44$ & Poor ( usually depart from natural or desirable level) \\
\hline
\end{tabular}

\section{Results and discussion}

The average of some of the physical and chemical properties (turbidity, alkalinity, and calcium) in all six sample locations during study period are greater than the permissible level recommended by the Iraqi standard for drinking water, respectively greater than $5 \mathrm{NTU}, 125 \mathrm{mg} /$ $\mathrm{L}$, and $100 \mathrm{mg} / \mathrm{L}$. Figure 2 shows the variation in turbidity values during study period in two locations samples in Kut city, while the sulfate values recorded are greater than recommended levels $(250 \mathrm{mg} / \mathrm{L})$ in all locations during some specific months. Figure 3 shows the variation of sulfate also in these two locations in kut city. The results obtained by this investigation showed that the values of other parameters are within Iraqi standard. See tables in appendix A, while the permissible levels for all water parameters in the study are tabulated in table 3 for Iraqi, WHO, and CCME.

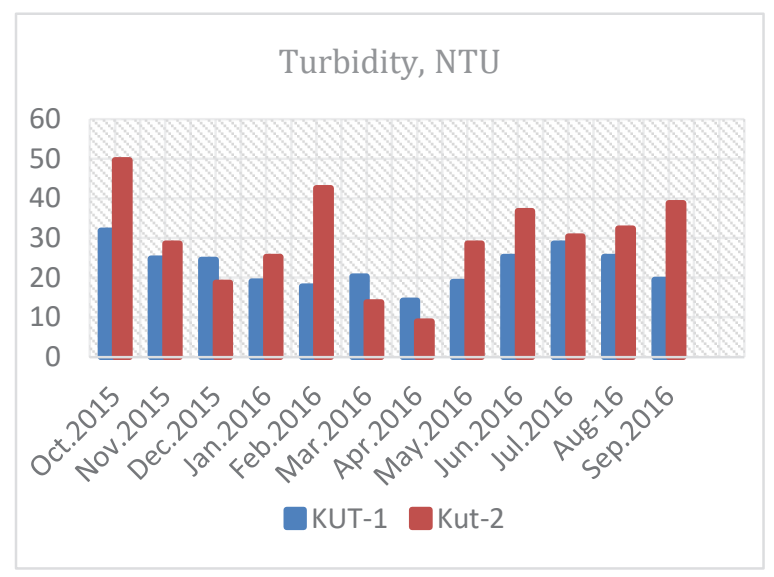

Fig.2. Variation of turbidity values in two locations samples in Kut City.

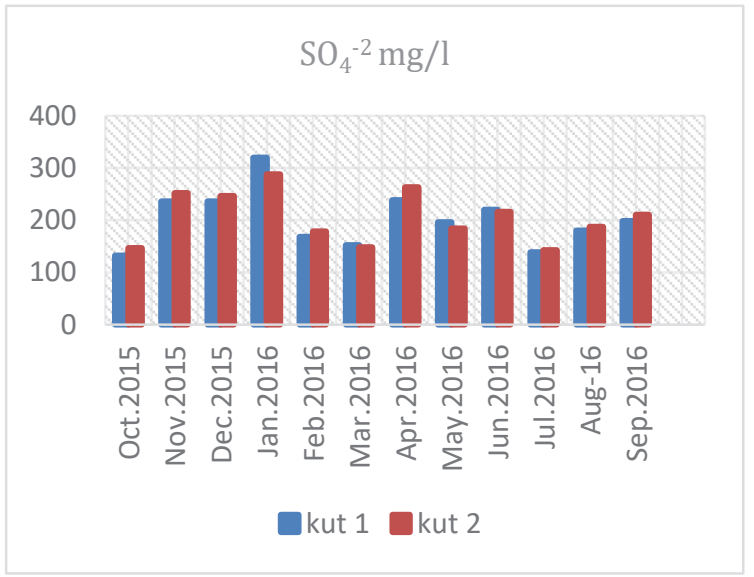

Fig.3. Shows the values of sulfate recorded during one year in two locations in Kut city 
Table 3. Shows the limits of some raw water parameters for Iraq, WHO, and CCME

\begin{tabular}{|c|c|c|c|c|c|c|c|c|c|c|c|c|}
\hline parameter & $\mathrm{pH}$ & $\begin{array}{l}\text { TDS } \\
\mathrm{mg} / \mathrm{l}\end{array}$ & $\begin{array}{c}\text { Turbidit } \\
\mathrm{y} \\
\text { (NTU) }\end{array}$ & $\begin{array}{l}\text { Alkalini } \\
\text { ty } \mathrm{mg} / \mathrm{l}\end{array}$ & $\begin{array}{c}\text { Nitrate } \\
\mathrm{mg} / \mathrm{l}\end{array}$ & $\begin{array}{l}\text { T.H as } \\
\mathrm{caco}_{3}\end{array}$ & $\begin{array}{l}\mathrm{Ca}^{+2} \\
\mathrm{mg} / 1\end{array}$ & $\begin{array}{c}\mathrm{Mg} \\
\mathrm{mg} / 1\end{array}$ & $\mathrm{~K} \mathrm{mg/l}$ & $\mathrm{Na} \mathrm{mg/l}$ & $\begin{array}{c}\mathrm{Cl} \\
\mathrm{mg} / \mathrm{L}\end{array}$ & $\begin{array}{c}\mathrm{SO}_{4} \\
\mathrm{mg} / \mathrm{L}\end{array}$ \\
\hline $\begin{array}{c}\text { Iraqi } \\
\text { standard }\end{array}$ & $\begin{array}{l}6.5- \\
8.5\end{array}$ & 1000 & 5 & 125 & 50 & 500 & 50 & 50 & 10 & 200 & 250 & 250 \\
\hline WHO & $\begin{array}{l}6.5- \\
9.5\end{array}$ & 500 & 5 & 100 & 50 & 250 & 100 & 50 & 12 & 200 & 250 & 250 \\
\hline CCME & $\begin{array}{l}6.5- \\
9.0\end{array}$ & 500 & 5 & - & 48.2 & 250 & - & 50 & 12 & 100 & 110 & 125 \\
\hline
\end{tabular}

The estimated values of water quality index by CCME method for all locations are tabulated in table 4, the values are ranged between 70.4 and 72.0 .

The water quality of Tigris river and its branches( Al- Djila and Ghraff )in Wassite governorate are ranged in fair class ( 65-79), that means the river required to be managed, and take care to be under consideration to control the source of pollutant that thrown to the river to keep them in its natural conditions.

Table 4. Shows the values of CCME WQI for raw water in Wassite governorate.

\begin{tabular}{|l|c|c|c|c|}
\hline location & F1 & F2 & F3 & CCMEWQI \\
\hline Al-Swaira & 33.3 & 25.7 & 23.3 & 72.0 \\
\hline Al- Noamania & 33.3 & 25.7 & 27.5 & 70.8 \\
\hline Before Kut Barrage & 33.3 & 25.7 & 27.0 & 71.1 \\
\hline After Kut Barrage & 33.3 & 27.0 & 27.0 & 70.4 \\
\hline Al-Djiala river & 33.3 & 25.7 & 28.0 & 70.8 \\
\hline Al-Gharaff river & 33.3 & 25.7 & 27.0 & 70.5 \\
\hline
\end{tabular}

\section{Conclusions}

Water quality index is a useful tools for the assessment of raw water states and can be used in formulating the pollution control strategies in terms of required treatment.

The calculated water quality index results showed that the Tigris raw water quality in Wassit governorate in all six sampling location were in fair condition with accordance to CCME WQI categories.

The raw water of Tigris river in Wassite governorate required to be observed and managed well by the local authorities to keep it in its natural condition and prevent degradation of its properties.

\section{References}

1- CCME, Canadian Council of Ministers of the Environment (2001). Water Quality Index 1.0
Technical Report". In Canadian Environmental Quality Guidelines.

2- S. Alhashimi, A. Mustafa. International Journal of Environment and Water (2012)

3- H. Ismail. J. Al-Taqani, vol.26, no. 2. (2013)

4- L. Abdulkareem. J. Appl. Science, Engineering and Technology10(11)- 1343-1346 (2015)

5- S. Ewaid. J. of Science, Vol. 57, No. 24, pp: 878-885(2016)

6- K. Husain, J. Environmental Monitoring and Assessment, 88: 221-242 (2003).

7- UNEP/GEMS,. http://www.gemswater.org/.

8- L. Boemo, A.Musso. j. Water SA., 33(4): 479485 (2007)

9- S. Gholamreza , A. Masoud, j. Environmental Science, 6, (4):19-28(2009).

10- A. Al-Obaidy. J. Water Resource and Protection, 2: 629-635(2010)

11- Z. Al-janabi, j. Al-Nahrain Univ., 15(1) 119126(2012)

12- Word Health Organization (WHO)," Guidelines for Drinking Water Quality " $4^{\text {th }}$ Edition Word Health Organization ( WHO), Geneva(2011) 


\section{APPENDIX A}

Table A1. Chemical test results for raw water at Swiara city station.

\begin{tabular}{|c|c|c|c|c|c|c|c|c|c|c|c|c|}
\hline parameter & $\mathrm{pH}$ & $\begin{array}{c}\mathrm{TDS} \\
\mathrm{mg} / 1\end{array}$ & $\begin{array}{c}\text { Turbidit } \\
\mathrm{y} \\
\mathrm{NTU})\end{array}$ & $\begin{array}{c}\text { Alkalinit } \\
\mathrm{y} \mathrm{mg} / \mathrm{l}\end{array}$ & $\begin{array}{c}\text { Nitrate } \\
\mathrm{mg} / \mathrm{l}\end{array}$ & $\begin{array}{c}\mathrm{T} . \mathrm{H} \\
\mathrm{as} \\
\mathrm{caco} \\
3\end{array}$ & $\begin{array}{c}\mathrm{Ca}^{+2} \\
\mathrm{mg} / \mathrm{l}\end{array}$ & $\begin{array}{c}\mathrm{Mg} \\
\mathrm{mg} / 1\end{array}$ & $\begin{array}{c}\mathrm{K} g \\
\mathrm{mg} / \\
1\end{array}$ & $\begin{array}{c}\mathrm{Na} \\
\mathrm{mg} / 1\end{array}$ & $\begin{array}{c}\mathrm{Cl} \\
\mathrm{mg} / \mathrm{L}\end{array}$ & $\begin{array}{c}\mathrm{SO}_{4} \\
\mathrm{mg} / \mathrm{L}\end{array}$ \\
\hline Oct.2015 & 7.31 & 684 & 16 & 152 & 4.1 & 286 & 74 & 25 & 4.21 & 86.8 & 107.8 & 129 \\
\hline Nov.2015 & 7.26 & 824 & 29.2 & 160 & 5.4 & 324 & 88 & 25 & 5.2 & 105 & 137.2 & 240 \\
\hline Dec.2015 & 7.06 & 786 & 18.1 & 150 & 4.6 & 316 & 80.6 & 26 & 3.8 & 105.5 & 132.3 & 216 \\
\hline Jan.2016 & 7.21 & 864 & 14.9 & 180 & 4.6 & 356 & 88.2 & 34 & 4.2 & 120.5 & 135.2 & 336 \\
\hline Feb.2016 & 7.76 & 636 & 9.44 & 150 & 5.4 & 290 & 72 & 24.2 & 4.3 & 98.5 & 117.6 & 162 \\
\hline Mar.2016 & 7.15 & 602 & 14.4 & 146 & 4.2 & 308 & 70.4 & 28.8 & 3.2 & 92.5 & 109.7 & 173 \\
\hline Apr.2016 & 7.62 & 696 & 19.7 & 156 & 3.2 & 286 & 67.4 & 30.2 & 3.2 & 90 & 123.5 & 208 \\
\hline May.2016 & 7.6 & 522 & 23.6 & 140 & 3.6 & 286 & 72 & 24 & 3.4 & 86.4 & 107.8 & 176 \\
\hline Jun.2016 & 7.36 & 630 & 17.7 & 150 & 4.2 & 286 & 80.4 & 21.2 & 3.1 & 86.5 & 111.7 & 200 \\
\hline Jul.2016 & 7.6 & 590 & 31.5 & 152 & 4.4 & 280 & 73.2 & 25 & 3.4 & 95 & 113.7 & 132 \\
\hline Aug 2016 & 7.73 & 596 & 32.4 & 156 & 3.4 & 274 & 75 & 20.8 & 4.2 & 80.5 & 107.8 & 162 \\
\hline Sep.2016 & 7.2 & 658 & 10.2 & 166 & 3.4 & 310 & 82 & 26.6 & 2.8 & 95.5 & 129.3 & 200 \\
\hline objective & $6.5-$ & 1000 & 5 & 125 & 50 & 500 & 50 & 50 & 10 & 200 & 250 & 250 \\
\hline
\end{tabular}

$\mathrm{F} 1=33.3, \mathrm{~F} 2=25.7, \mathrm{~F} 3=23.3, \mathbf{W Q I}=\mathbf{7 2}$

Table A2. Chemical test results for raw water at Noamania city station.

\begin{tabular}{|l|l|l|l|l|l|l|l|l|l|l|l|l|}
\hline parameter & $\mathrm{pH}$ & $\begin{array}{c}\mathrm{TDS} \\
\mathrm{mg} / 1\end{array}$ & $\begin{array}{c}\text { Turbidity } \\
\text { (NTU) }\end{array}$ & $\begin{array}{c}\text { Alkalinity } \\
\mathrm{mg} / 1\end{array}$ & $\begin{array}{c}\text { Nitraite } \\
\mathrm{mg} / 1\end{array}$ & $\begin{array}{c}\mathrm{T} . \mathrm{H} \\
\mathrm{as} \\
\mathrm{cac0} 3\end{array}$ & $\begin{array}{c}\mathrm{Ca}^{2} \\
\mathrm{mg} / 1\end{array}$ & $\begin{array}{c}\mathrm{Mg} \\
\mathrm{mg} / 1\end{array}$ & $\begin{array}{c}\mathrm{K} \\
\mathrm{mg} / 1\end{array}$ & $\begin{array}{c}\mathrm{Na} \\
\mathrm{mg} / 1\end{array}$ & $\begin{array}{c}\mathrm{Cl} \\
\mathrm{mg} / \mathrm{L}\end{array}$ & $\begin{array}{c}\mathrm{SO}_{4} \\
\mathrm{mg} / \mathrm{L}\end{array}$ \\
\hline Oct.2015 & 7.29 & 710 & 27.8 & 160 & 2.84 & 300 & 78 & 23.5 & 5.16 & 92 & 113 & 140 \\
\hline Nov.2015 & 7.22 & 820 & 23.6 & 166 & 5.8 & 320 & 86 & 28 & 5.6 & 115 & 139 & 248 \\
\hline Dec.2015 & 7.08 & 792 & 16.8 & 165 & 4.2 & 328 & 83.4 & 27.8 & 3.4 & 110 & 135 & 224 \\
\hline Jan.2016 & 7.32 & 860 & 20.6 & 170 & 5.2 & 340 & 86.2 & 36 & 4.8 & 115 & 137 & 314 \\
\hline Feb.2016 & 7.81 & 654 & 40.2 & 156 & 5.8 & 298 & 74.2 & 26.3 & 4.8 & 105 & 124 & 148 \\
\hline Mar.2016 & 7.32 & 674 & 13.5 & 160 & 4.4 & 316 & 72 & 28.2 & 3.8 & 98 & 115 & 162 \\
\hline Apr.2016 & 7.7 & 796 & 23.8 & 160 & 4.8 & 300 & 71 & 28.6 & 3.4 & 96 & 127 & 227 \\
\hline May.2016 & 7.4 & 564 & 21.8 & 156 & 3.4 & 300 & 80 & 23.4 & 4.1 & 91.5 & 115 & 166 \\
\hline Jun.2016 & 7.57 & 710 & 20.3 & 170 & 4.6 & 300 & 80 & 23.5 & 3.4 & 95 & 121 & 183 \\
\hline Jul.2016 & 7.4 & 610 & 36.8 & 150 & 4.8 & 294 & 78.6 & 23.6 & 3.6 & 98.5 & 119 & 128 \\
\hline Aug 2016 & 7.81 & 630 & 26.7 & 160 & 3.6 & 286 & 78 & 22.5 & 3.4 & 85.2 & 117 & 172 \\
\hline Sep.2016 & 7.6 & 666 & 32.6 & 172 & 3.2 & 320 & 86 & 25 & 3.2 & 90.5 & 132 & 186 \\
\hline objective & $6.5-$ & 1000 & 5 & 125 & 50 & 500 & 50 & 50 & 50 & 200 & 250 & 250 \\
\hline
\end{tabular}

$\mathrm{F} 1=33.3, \mathrm{~F} 2=25.69 \mathrm{~F} 3=27.53, \mathbf{W Q I}=\mathbf{7 0 . 9 7}$

Table A3. Chemical test results for raw water at Kut city station. Before Kut Barrage section 1. 


\begin{tabular}{|l|l|l|l|l|l|l|l|l|l|l|l|l|}
\hline parameter & $\mathrm{pH}$ & $\begin{array}{c}\mathrm{TDS} \\
\mathrm{mg} / 1\end{array}$ & $\begin{array}{c}\text { Turbidity } \\
\text { (NTU) }\end{array}$ & $\begin{array}{c}\text { Alkalinity } \\
\mathrm{mg} / \mathrm{l}\end{array}$ & $\begin{array}{c}\text { Nitrite } \\
\mathrm{mg} / \mathrm{l}\end{array}$ & $\begin{array}{c}\mathrm{T} . \mathrm{H} \\
\mathrm{as} \\
\mathrm{cac0} 3\end{array}$ & $\begin{array}{c}\mathrm{Ca}+2 \\
\mathrm{mg} / 1\end{array}$ & $\begin{array}{c}\mathrm{Mg} \\
\mathrm{mg} / 1\end{array}$ & $\begin{array}{c}\mathrm{K} \\
\mathrm{mg} / 1\end{array}$ & $\begin{array}{c}\mathrm{Na} \\
\mathrm{mg} / 1\end{array}$ & $\begin{array}{c}\mathrm{Cl} \\
\mathrm{mg} / \mathrm{L}\end{array}$ & $\begin{array}{c}\mathrm{SO}_{4} \\
\mathrm{mg} / \mathrm{L}\end{array}$ \\
\hline Oct.2015 & 7.3 & 692 & 31.8 & 146 & 2.84 & 294 & 80 & 21.4 & 3.82 & 90.5 & 107.8 & 132 \\
\hline Nov.2015 & 7.2 & 826 & 24.8 & 178 & 5.2 & 336 & 91 & 30 & 4.8 & 98.8 & 133.3 & 236 \\
\hline Dec.2015 & 7.04 & 826 & 24.5 & 160 & 4.8 & 310 & 84 & 22.6 & 4.2 & 100.8 & 139 & 236 \\
\hline Jan.2016 & 7.64 & 986 & 19 & 200 & 6.8 & 380 & 98 & 38.4 & 5.6 & 138.6 & 152 & 320 \\
\hline Feb.2016 & 7.58 & 720 & 17.8 & 148 & 4.6 & 300 & 75.6 & 30 & 3.6 & 96.4 & 113.7 & 168 \\
\hline Mar.2016 & 7.23 & 620 & 20.3 & 155 & 5.2 & 294 & 68.7 & 30 & 4.6 & 105 & 120.5 & 152 \\
\hline Apr.2016 & 7.62 & 824 & 14.2 & 150 & 3.6 & 290 & 68.6 & 30 & 4.4 & 100.5 & 122.5 & 238 \\
\hline May.2016 & 7.8 & 618 & 18.9 & 144 & 2.8 & 282 & 77.4 & 26.4 & 3.2 & 85.5 & 109.8 & 196 \\
\hline Jun.2016 & 7.42 & 626 & 25.2 & 160 & 3.8 & 284 & 76 & 22.4 & 2.8 & 100 & 115.6 & 220 \\
\hline Jul.2016 & 7.5 & 616 & 28.6 & 166 & 3.8 & 266 & 70.6 & 21.8 & 4.2 & 105 & 127.4 & 138 \\
\hline Aug 2016 & 7.9 & 664 & 25.2 & 156 & 4.2 & 264 & 74.2 & 21.2 & 3.6 & 90.5 & 125.4 & 180 \\
\hline Sep.2016 & 7.4 & 654 & 19.4 & 160 & 4.4 & 300 & 80.2 & 27.5 & 2.8 & 100 & 127.4 & 198 \\
\hline objective & $6.5-$ & 1000 & 5 & 125 & 50 & 500 & 50 & 50 & 50 & 200 & 250 & 250 \\
\hline
\end{tabular}

$\mathrm{F} 1=33.33, \mathrm{~F} 2=25.7, \mathrm{~F} 3=26.84, \mathbf{W Q I}=\mathbf{7 1 . 1 9}$

Table A4. Chemical test results for raw water at Kut City station. Section 2. After Kut Barrage

\begin{tabular}{|c|c|c|c|c|c|c|c|c|c|c|c|c|}
\hline parameter & $\mathrm{pH}$ & $\begin{array}{l}\mathrm{TDS} \\
\mathrm{mg} / \mathrm{l}\end{array}$ & $\begin{array}{c}\text { Turbidit } \\
y \\
\text { (NTU) }\end{array}$ & $\begin{array}{c}\text { Alkalinit } \\
\text { y mg/l }\end{array}$ & $\begin{array}{c}\text { Nitrate } \\
\mathrm{mg} / 1 \\
\mathrm{NO3}\end{array}$ & $\begin{array}{c}\text { T.H } \\
\text { as } \\
\text { caco } \\
3\end{array}$ & $\begin{array}{l}\mathrm{Ca}^{+2} \\
\mathrm{mg} / \mathrm{l}\end{array}$ & $\begin{array}{l}\mathrm{Mg}^{+2} \\
\mathrm{mg} / \mathrm{l}\end{array}$ & $\begin{array}{c}\mathrm{K}^{+1} \\
\mathrm{mg} / \\
\mathrm{l}\end{array}$ & $\begin{array}{l}\mathrm{Na}^{+1} \\
\mathrm{mg} / \mathrm{l}\end{array}$ & $\begin{array}{c}\mathrm{Cl}^{-1} \\
\mathrm{mg} / \mathrm{L}\end{array}$ & $\begin{array}{l}\mathrm{SO}_{4}^{-2} \\
\mathrm{mg} / \mathrm{L}\end{array}$ \\
\hline Oct.2015 & 7.28 & 718 & 49.6 & 166 & 3.66 & 310 & 84 & 22 & 4.81 & 98.8 & 122 & 146 \\
\hline Nov.2015 & 7.19 & 832 & 28.6 & 184 & 6.6 & 342 & 90 & 32 & 6.4 & 126 & 147 & 252 \\
\hline Dec.2015 & 7.11 & 818 & 18.7 & 156 & 5.6 & 330 & 90 & 28 & 4.8 & 120 & 143 & 246 \\
\hline Jan.2016 & 7.52 & 876 & 25.2 & 176 & 4.8 & 336 & 96 & 36 & 5.2 & 125 & 139 & 288 \\
\hline Feb.2016 & 7.78 & 766 & 42.6 & 166 & 5.8 & 312 & 80 & 28 & 4.8 & 115 & 131 & 178 \\
\hline Mar.2016 & 7.08 & 604 & 13.8 & 166 & 4.8 & 310 & 71.5 & 29 & 3.8 & 100 & 117 & 148 \\
\hline Apr.2016 & 7.84 & 838 & 8.91 & 166 & 5.2 & 310 & 72 & 31 & 4.2 & 110 & 135 & 263 \\
\hline May.2016 & 7.5 & 632 & 28.6 & 160 & 4.6 & 318 & 82 & 24.6 & 4.6 & 105 & 121 & 184 \\
\hline Jun.2016 & 7.64 & 642 & 36.8 & 170 & 4.2 & 296 & 81 & 21.6 & 3.2 & 105 & 125 & 216 \\
\hline Jul.2016 & 7.7 & 622 & 30.4 & 170 & 4.2 & 290 & 75 & 25 & 4.8 & 110 & 125 & 142 \\
\hline Aug 2016 & 7.93 & 668 & 32.4 & 166 & 3.8 & 276 & 71.5 & 23 & 3.2 & 95 & 129 & 187 \\
\hline Sep.2016 & 7.7 & 686 & 38.8 & 180 & 3.8 & 328 & 82 & 28.8 & 4.2 & 105 & 137 & 210 \\
\hline objective & $\begin{array}{l}6.5- \\
8.5\end{array}$ & 1000 & 5 & 125 & 50 & 500 & 50 & 50 & 50 & 200 & 250 & 250 \\
\hline
\end{tabular}

$\mathrm{F} 1=33.33, \mathrm{~F} 2=27, \mathrm{~F} 3=27, \mathbf{W Q I}=\mathbf{7 0 . 4 2}$ 
Table A5. Chemical test results for raw water at Kut City station. Al- Dijialy section

\begin{tabular}{|l|l|l|l|l|l|l|l|l|l|l|l|l|}
\hline parameter & $\mathrm{pH}$ & $\begin{array}{l}\mathrm{TDS} \\
\mathrm{mg} / 1\end{array}$ & $\begin{array}{c}\text { Turbidity } \\
\text { (NTU) }\end{array}$ & $\begin{array}{c}\text { Alkalinity } \\
\mathrm{mg} / 1\end{array}$ & $\begin{array}{c}\text { Nitrate } \\
\mathrm{mg} / 1 \\
\mathrm{NO3}\end{array}$ & $\begin{array}{c}\mathrm{T} . \mathrm{H} \\
\mathrm{as} \\
\mathrm{cac0} 3\end{array}$ & $\begin{array}{l}\mathrm{Ca}^{+2} \\
\mathrm{mg} / 1\end{array}$ & $\begin{array}{l}\mathrm{Mg}^{+2} \\
\mathrm{mg} / \mathrm{l}\end{array}$ & $\begin{array}{l}\mathrm{K}^{+1} \\
\mathrm{mg} / \mathrm{l}\end{array}$ & $\begin{array}{l}\mathrm{Na}^{+1} \\
\mathrm{mg} / 1\end{array}$ & $\begin{array}{c}\mathrm{Cl}^{-1} \\
\mathrm{mg} / \mathrm{L}\end{array}$ & $\begin{array}{l}\mathrm{SO}^{-2} \\
\mathrm{mg} / \mathrm{L}\end{array}$ \\
\hline Oct.2015 & 7.25 & 734 & 30.4 & 180 & 2.85 & 330 & 82 & 30 & 3.6 & 100 & 128 & 156 \\
\hline Nov.2015 & 7.17 & 816 & 34.4 & 156 & 5.2 & 310 & 82 & 24 & 5.8 & 105 & 137 & 246 \\
\hline Dec.2015 & 7.14 & 828 & 21.4 & 160 & 5.3 & 328 & 88 & 25 & 3.6 & 115 & 140 & 228 \\
\hline Jan.2016 & 7.26 & 870 & 8.47 & 180 & 4.2 & 350 & 87 & 35 & 4.3 & 132 & 127 & 276 \\
\hline Feb.2016 & 7.43 & 732 & 8.4 & 160 & 4.2 & 308 & 75 & 28 & 3 & 109 & 123 & 172 \\
\hline Mar.2016 & 7.17 & 616 & 5.71 & 140 & 4.6 & 286 & 69 & 26 & 4.4 & 116 & 127 & 137 \\
\hline Apr.2016 & 7.72 & 882 & 6.51 & 150 & 4.2 & 326 & 80 & 32 & 3.2 & 92 & 124 & 221 \\
\hline May.2016 & 7.4 & 642 & 34.5 & 156 & 3.8 & 305 & 80 & 25 & 4.2 & 100 & 137 & 168 \\
\hline Jun.2016 & 7.64 & 746 & 15.4 & 176 & 5.1 & 310 & 82 & 26 & 4.1 & 116 & 129 & 250 \\
\hline Jul.2016 & 7.8 & 630 & 39.6 & 176 & 4.6 & 272 & 72 & 22 & 3.7 & 100 & 124 & 152 \\
\hline Aug 2016 & 7.84 & 660 & 16.3 & 150 & 3.2 & 292 & 80 & 22 & 3.4 & 87 & 131 & 176 \\
\hline Sep.2016 & 7.8 & 668 & 52.4 & 170 & 4.8 & 308 & 82 & 28 & 3.4 & 95 & 132 & 188 \\
\hline objective & $6.5-$ & 1000 & 5 & 125 & 50 & 500 & 50 & 50 & 50 & 200 & 250 & 250 \\
\hline
\end{tabular}

$\mathrm{F} 1=33.3, \mathrm{~F} 2=25.7, \mathrm{~F} 3=28, \mathbf{W Q I}=\mathbf{7 0 . 8 2}$

Table A6. Chemical test results for raw water at Kut City station. Al- ghraf section

\begin{tabular}{|c|c|c|c|c|c|c|c|c|c|c|c|c|}
\hline parameter & $\mathrm{pH}$ & $\begin{array}{l}\mathrm{TDS} \\
\mathrm{mg} / \mathrm{l}\end{array}$ & $\begin{array}{l}\text { Turbidit } \\
\mathrm{y} \\
\text { (NTU) }\end{array}$ & $\begin{array}{l}\text { Alkalinit } \\
\text { y mg/l }\end{array}$ & $\begin{array}{l}\text { Nitrate } \\
\mathrm{mg} / \mathrm{l} \\
\mathrm{NO} 3\end{array}$ & $\begin{array}{l}\text { T.H } \\
\text { as } \\
\text { caco } \\
3\end{array}$ & $\begin{array}{l}\mathrm{Ca}^{+2} \\
\mathrm{mg} / \mathrm{l}\end{array}$ & $\begin{array}{l}\mathrm{Mg}^{+2} \\
\mathrm{mg} / 1\end{array}$ & $\begin{array}{l}\mathrm{K}^{+1} \\
\mathrm{mg} / \\
1\end{array}$ & $\begin{array}{l}\mathrm{Na}^{+1} \\
\mathrm{mg} / 1\end{array}$ & $\begin{array}{l}\mathrm{Cl}^{-1} \\
\mathrm{mg} / \mathrm{L}\end{array}$ & $\begin{array}{l}\mathrm{SO}_{4}^{-2} \\
\mathrm{mg} / \mathrm{L}\end{array}$ \\
\hline Oct.2015 & 7.21 & 726 & 60 & 176 & 2.46 & 324 & 80 & 31.5 & 3.44 & 105 & 122.5 & 156 \\
\hline Nov.2015 & 7.16 & 808 & 32.6 & 148 & 5.4 & 300 & 84 & 21.4 & 5.4 & 100 & 135 & 240 \\
\hline Dec. 2015 & 7.08 & 842 & 26.2 & 170 & 5.8 & 340 & 92 & 26.5 & 4.4 & 110 & 137 & 248 \\
\hline Jan.2016 & 7.66 & 852 & 30.9 & 166 & 6.4 & 330 & 84 & 30.8 & 4.2 & 105 & 140 & 310 \\
\hline Feb.2016 & 7.52 & 728 & 19.6 & 155 & 4.8 & 296 & 70.6 & 30 & 3.8 & 110 & 127 & 184 \\
\hline Mar.2016 & 7.21 & 586 & 6.25 & 146 & 4.8 & 300 & 65 & 28.2 & 4.2 & 96.5 & 111 & 128 \\
\hline Apr.2016 & 7.82 & 834 & 21.8 & 146 & 3.6 & 296 & 70 & 31.5 & 3.5 & 95 & 119 & 250 \\
\hline May.2016 & 7.7 & 626 & 22.6 & 140 & 4.1 & 300 & 79 & 27.2 & 3.8 & 96 & 118 & 179 \\
\hline Jun.2016 & 7.72 & 660 & 43.6 & 180 & 5.4 & 268 & 80.5 & 26 & 4.2 & 110 & 132 & 210 \\
\hline Jul.2016 & 7.3 & 624 & 29.6 & 160 & 3.8 & 264 & 70.8 & 23.2 & 3.8 & 116 & 132 & 148 \\
\hline Aug 2016 & 7.82 & 666 & 28.7 & 152 & 4.2 & 320 & 72.8 & 22.4 & 3.2 & 88.5 & 122 & 178 \\
\hline Sep.2016 & 7.3 & 672 & 22.6 & 166 & 3.6 & 325 & 88 & 25.6 & 3.2 & 105 & 129 & 192 \\
\hline objective & $\begin{array}{l}6.5- \\
8.5\end{array}$ & 1000 & 5 & 125 & 50 & 500 & 50 & 50 & 50 & 200 & 250 & 250 \\
\hline
\end{tabular}

$\mathrm{F} 1=33.3, \mathrm{~F} 2=25.7, \mathrm{~F} 3=27, \mathrm{WQI}=70.5$ 


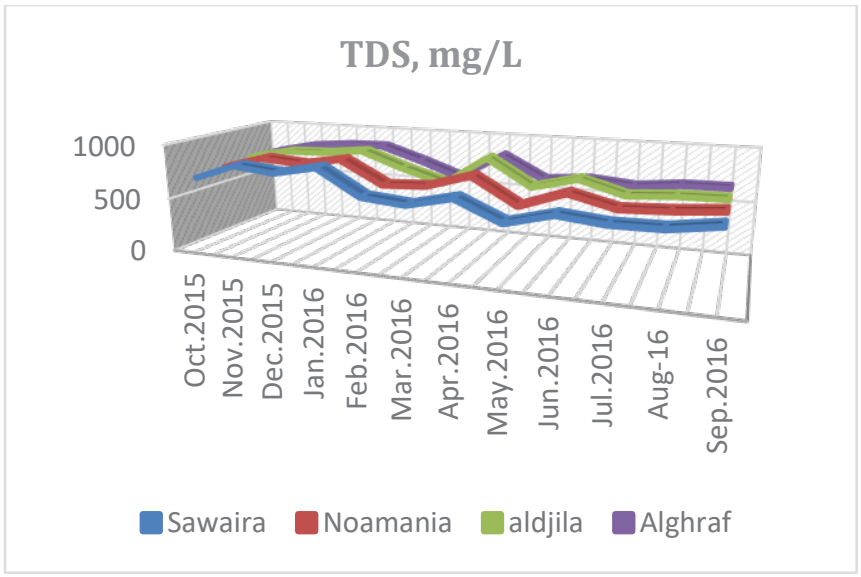

Fig. A1 Shows the variation of TDS in four location during study period. 